\title{
EyeRIS: A general-purpose system for eye-movement-contingent display control
}

\author{
Fabrizio Santini, Gabriel Redner, Ramon Iovin, and Michele Rucci \\ Boston University, Boston, Massachusetts
}

\begin{abstract}
In experimental studies of visual performance, the need often emerges to modify the stimulus according to the eye movements performed by the subject. The eye-movement-contingent display (EMCD) methodology enables accurate control of the position and motion of the stimulus on the retina. EMCD procedures have been used successfully in many areas of vision science, including studies of visual attention or eye movements and physiological characterization of neuronal response properties. Unfortunately, the difficulty of real-time programming and the unavailability of flexible and economical systems that can be easily adapted to the diversity of experimental needs and laboratory setups have prevented the widespread use of EMCD control. This article describes EyeRIS, a general-purpose system for performing EMCD experiments on a Windows computer. Based on a digital signal processor with analog and digital interfaces, this integrated hardware and software system is responsible for sampling and processing oculomotor signals and subject responses and for modifying the stimulus displayed on a CRT according to a gaze-contingent procedure specified by the experimenter. EyeRIS is designed to update the stimulus with a delay of only $10 \mathrm{msec}$. To thoroughly evaluate EyeRIS's performance, this study was designed to (1) examine the response of the system in a number of EMCD procedures and computational benchmarking tests; (2) compare the accuracy of implementation of one particular EMCD procedure, retinal stabilization, with that produced by a standard tool used for this task; and (3) examine EyeRIS's performance in one of the many EMCD procedures that cannot be executed by means of any other currently available device.
\end{abstract}

Our eyes are always in motion. During natural viewing, saccades occur every few hundred milliseconds. Even between saccades, when the eyes are fixating on a target, small fixational eye movements, of which we are usually not aware, continually move the image of the scene on the retina (see Figure 1). It is surprising that the visual system is able to construct a coherent percept from such fragmentary and continuously changing input. Although much progress has been made in understanding how the brain processes sensory signals, understanding of the fundamental mechanisms by which visual information is organized into a global representation of a scene remains elusive.

In experimental studies of visual functions, it is often necessary to modify the stimulus according to the subject's eye movements. Eye-movement-contingent display (EMCD) control is a methodology that enables accurate control of the position and motion of the stimulus on the retina (Ditchburn \& Ginsborg, 1952; McConkie, 1997; Pritchard, 1961; Riggs \& Ratliff, 1952). It has been successfully used in many areas of vision science, ranging from the study of visual attention (Geisler \& Perry, 1998; Pomplun, Reingold, \& Shen, 2001b; Rayner, 1997) to the physiological characterization of neuronal response properties (Gur \& Snodderly, 1987; Snodderly, Kagan, \& Gur, 2001). In addition to its application in basic vision research, EMCD control is also crucial for the development of a variety of applications, such as augmented informa- tion displays (Nikolov et al., 2004; Reingold, Loschky, McConkie, \& Stampe, 2003) and image enhancement aids for the visually impaired (Peli \& Peli, 1984; Tang, Kim, \& Peli, 2004).

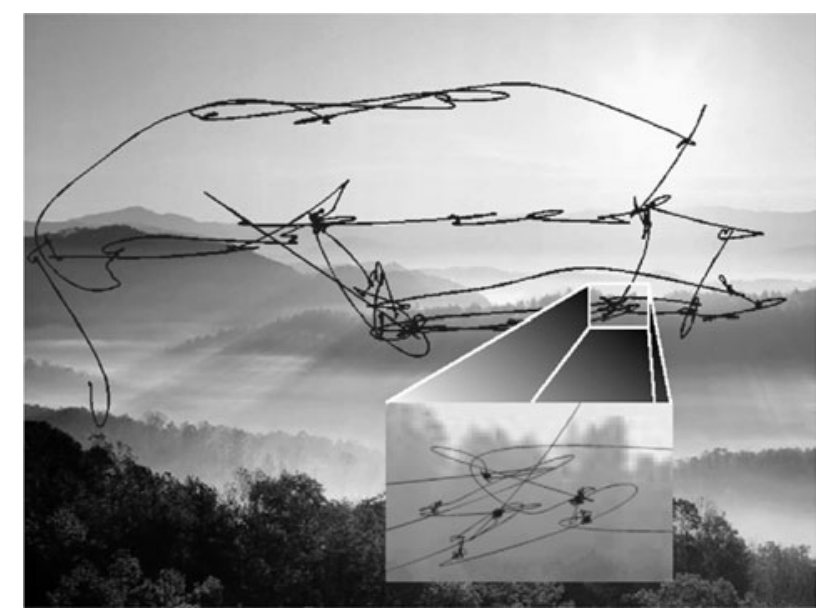

Figure 1. Example of oculomotor activity during unconstrained viewing of a natural scene. A trace of eye movements recorded by a dual-Purkinje-image eyetracker is shown superimposed on the original image. The inset panel shows an enlarged portion of the trace in which small fixational eye movements are present. The image was examined for a period of 30 sec. 
Figure 2 illustrates four examples of the application of EMCD control in different areas of vision science. In Figure 2A, the scene is resampled with space-variant resolution relative to the point of fixation, so as to replicate the eccentricity-dependent resolving power of the primate visual system (Geisler \& Perry, 1998; Perry \& Geisler, 2002). This kind of experiment, in which regions of a scene are dynamically modified according to the position of gaze, are useful for studying visual attention and quantifying the variables that determine saliency. Figure 2B shows the simulation of a visual scotoma (Bertera, 1988; Toufaili, Seibel,
\& McIntyre, 2004). A programmable EMCD system enables the simulation of many types of visual impairments, and thus finds useful application in medical evaluations of visual performance, patient rehabilitation, and the training of ophthalmologists. EMCD control also provides a powerful tool for improving the quality of neurophysiological recordings in alert and behaving subjects (Gur, Beylin, \& Snodderly, 1997). The physiological characterization of neuronal responses in alert subjects must overcome the challenge of unpredictable shifts in the locations of cell receptive fields caused by oculomotor activity (Leopold
A

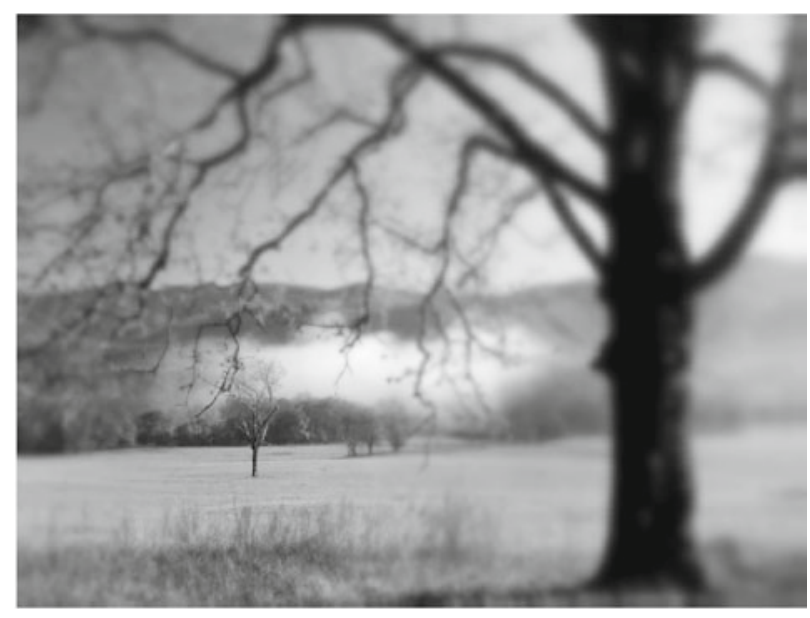

C

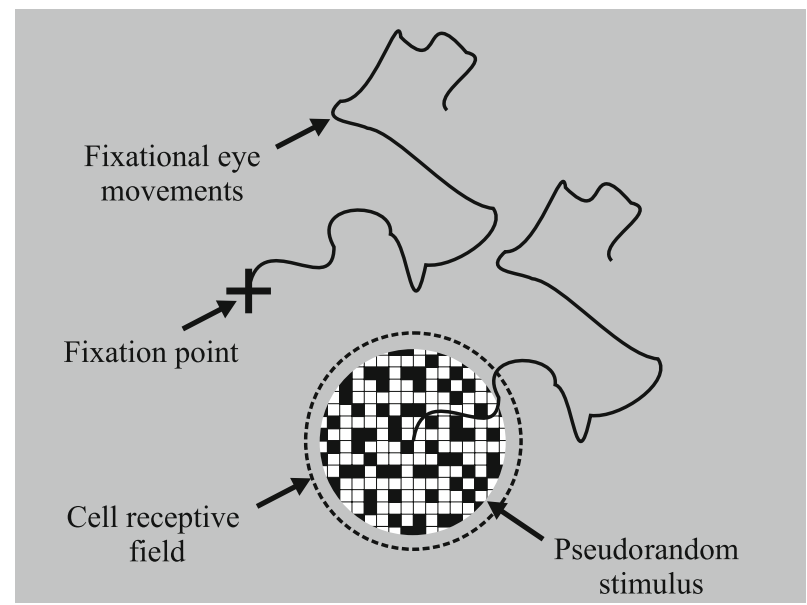

B

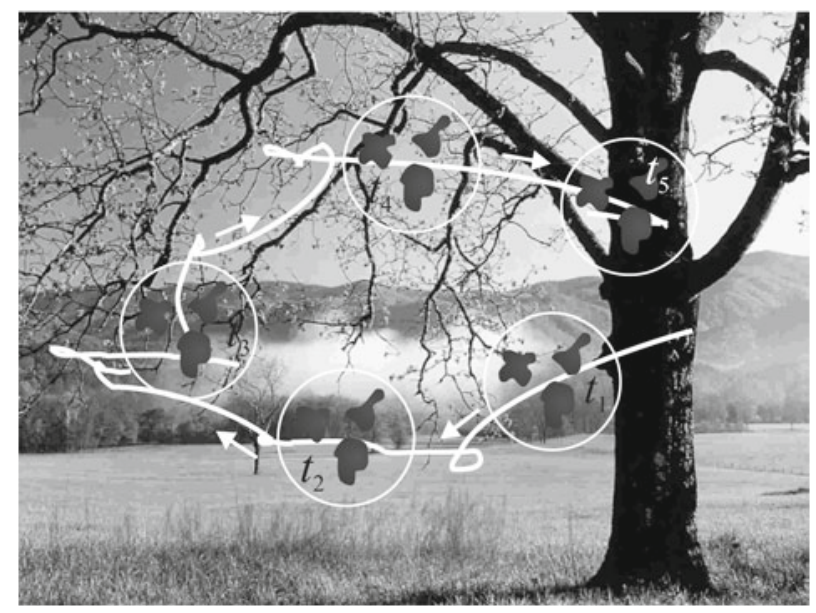

D

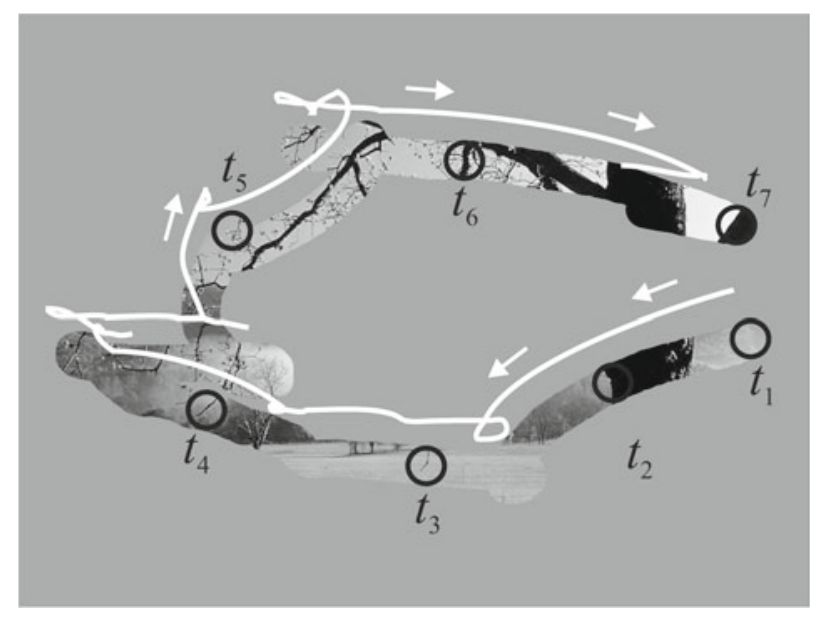

Figure 2. Four examples of EMCD experiments. (A) Real-time space-variant sampling. Varying the resolution of the image to follow the decrement in visual acuity with eccentricity is useful in studies of visual acuity and attention. (B) Simulation of a scotoma. The dark spots on the image follow the trajectory of the eye (white line) to simulate impaired regions of the visual field. Each circle shows the scotomas at a different time $t_{i}$. (C) Application of retinal stabilization in neurophysiology.A cell is stimulated with a pseudorandom spatiotemporal signal, as in reverse-correlation experiments. EMCD control enables the stimulus to follow the cell receptive field as it moves with the physiological instability of visual fixation. (D) Physiological characterization of retinal and extraretinal influences on cell responses. In the first phase of the experiment, cell responses are recorded during a sequence of guided eye movements in which the subject follows a fixation dot moving over the scene. During eye movements, the stimulus is presented only in the area covered by the cell receptive field, and pixels outside this area are dynamically set to a uniform gray. In the second phase, cell responses are recorded during presentations of the same spatiotemporal stimulus experienced by the cell in Phase 1 (the movie obtained by moving the aperture following the eye movements) under conditions of retinal stabilization. Comparison between Phases 1 and 2 allows characterization of proprioceptive influences. Control of the dimension of the stimulus window enables determination of influences from the classical and the extraclassical receptive field. 
\& Logothetis, 1998; Martinez-Conde, Macknik, \& Hubel, 2000; Snodderly et al., 2001).

As illustrated in Figure 2C, EMCD control enables accurate positioning of the stimulus in the receptive field of a recorded cell, despite fixational jitter. This technique has been shown to provide characterization of cell response properties in alert monkeys with a level of accuracy comparable to that obtainable under anesthesia (Gur \& Snodderly, 1987). Furthermore, as shown in Figure 2D, EMCD control can also be used to identify and isolate influences on neuronal responses originating from different sources, such as contributions from the classical and the extraclassical receptive field, as well as extraretinal modulations.

Despite the important benefits offered by EMCD control to many areas of vision research, a number of factors have prevented widespread use of this technique. A major limiting factor has been the unavailability of generalpurpose systems that enable flexible gaze-contingent manipulation of the stimulus.

Although several EMCD procedures are possible, the range of applications enabled by current systems remains minimal. A second limiting factor has been the difficulty of guaranteeing real-time performance - that is, ensuring an upper boundary on the delay between the occurrence of oculomotor events and update of the stimulus on the display. Real-time control generally requires advanced hardware development and programming expertise, which is not always available in vision science laboratories.

The tremendous improvements in computational power, video hardware, and eyetracking technologies of recent years, however, have opened the way to a flexible and economical approach to EMCD experiments. Digital signal processor (DSP) boards have dramatically increased in speed and can now be easily programmed and interfaced with other systems according to user demands. High-quality CRTs with refresh rates up to $200 \mathrm{~Hz}$ and video cards with built-in accelerators for the fast generation of visual stimuli are now available. The short delays and high quality of visualization provided by these systems are adequate for many experiments in visual neuroscience. Moreover, improvements in eyetracking technology have greatly enlarged the circle of potential users by

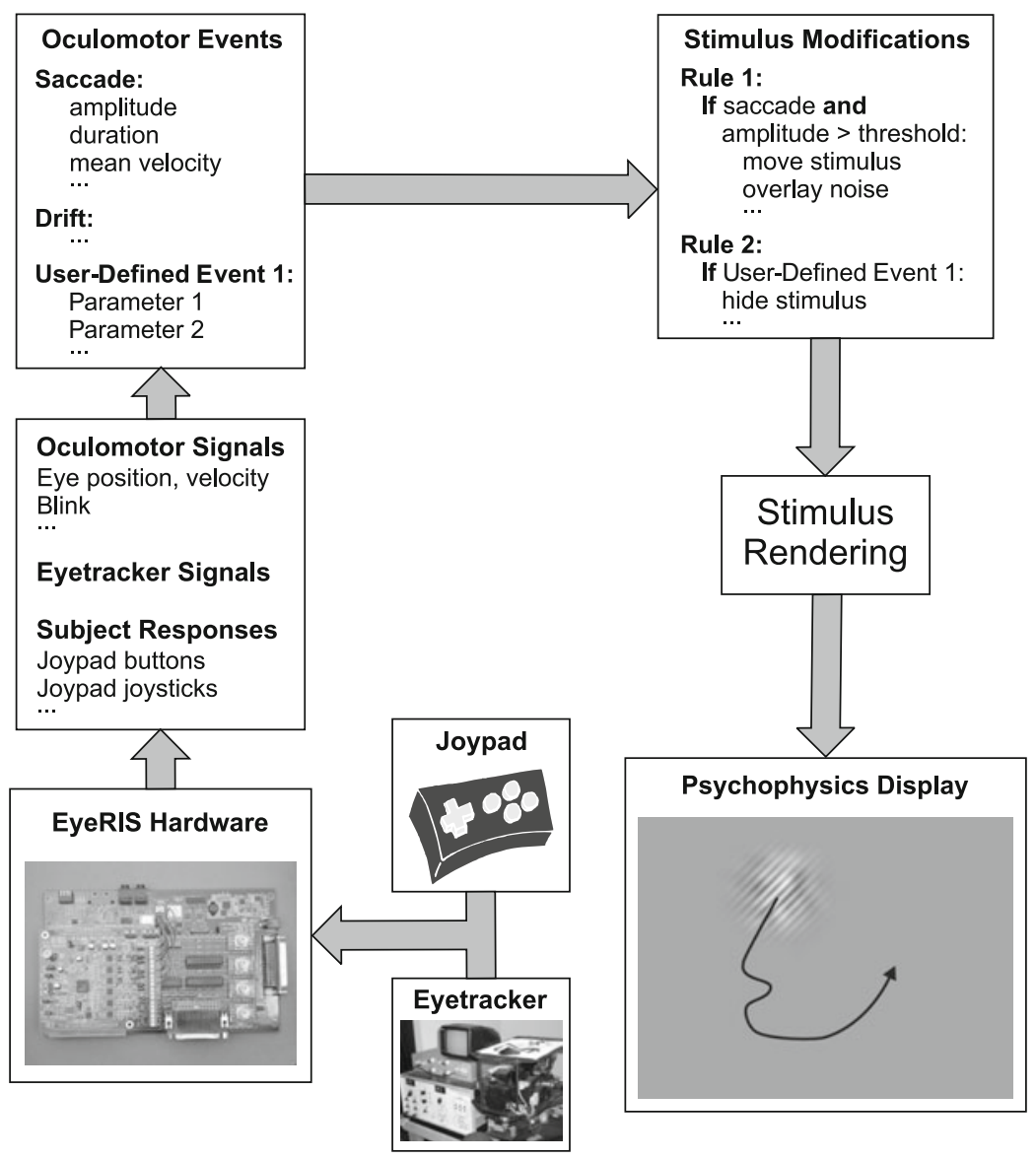

Figure 3. EyeRIS general architecture. The path by which data flow through the system is illustrated: Oculomotor signals from the eyetracker and subject responses are sampled and processed by a dedicated board. Kinematic signals are extracted and combined in real time to identify oculomotor events and their parameters, which are linked to gaze-contingent modifications of the stimulus following the rules specified by the experimenter. The resulting modifications are then displayed on the psychophysics monitor upon the subsequent screen refresh. 
miniaturizing the devices, significantly simplifying their use, and reducing their cost.

This article describes EyeRIS (Eye movement Realtime Integrated System), a general-purpose system for EMCD control. Building upon the recent technological advances, this system enables flexible design of EMCD experiments with a maximum delay of $10 \mathrm{msec}$. EyeRIS combines power, affordability, and simplicity of use. It works under Windows, the most commonly used operating system among researchers in neurophysiology and psychophysics. EyeRIS's hardware is composed of standard, commercially available signal processing boards, and its software is freely available on the Internet under an open-source license.

The remainder of this article is organized as follows: In the next section, we describe the main hardware and software components of EyeRIS. The Results section then contains reports about system performance on a variety of experimental tasks. Finally, the article concludes with a brief discussion of the experimental results and potential applications of the system.

\section{SYSTEM ARCHITECTURE}

Figure 3 gives an overview of the functional organization of EyeRIS. EyeRIS's inputs are the eye movement signals measured by an eyetracker and the subject's responses recorded by a joypad. These data are collected by the system's dedicated hardware, a DSP-based board equipped with both analog and digital inputs, and processed in real time to extract the fundamental kinematic signals of oculomotor activity. These signals are processed further and combined according to predefined rules in order to identify basic categories of oculomotor events (e.g., saccade, drift) and to evaluate their relevant parameters (e.g., saccade amplitude, mean drift velocity). The experimenter can modify these classification rules and thresholds to match experimental requirements. Furthermore, the experimenter can arbitrarily combine oculomotor signals, events, and the status of joypad buttons to create new userdefined events of relevance to the task at hand (e.g., a saccade with amplitude constrained to a specified range). All oculomotor variables and events are continually transmitted to the host PC, where they are associated with conditions and functions of stimulus manipulation according to the experimenter's specifications. Stimuli are visualized on the psychophysics CRT monitor and can be modified in real time according to any of the oculomotor variables and events produced by the system. Gaze-contingent display is designed to occur within a maximum delay equal to the refreshing of two frames on the CRT monitor. That is, with a refresh rate of $200 \mathrm{~Hz}$, the update of the stimulus on the screen occurs within $10 \mathrm{msec}$ (typical delay, $7.5 \mathrm{msec}$ ). In the rare event that this maximum delay is exceeded, an error signal is automatically generated by the system and the experimental trial in which the error occurred is flagged for the experimenter, so that it can be discarded during data analysis.

The following subsections describe the major components of EyeRIS in more detail. The DSP-board hardware and firmware are described first, followed by the software library running on the host PC, and an overview of the system timing and its real-time compliance.

\section{DSP Hardware}

The EyeRIS hardware is built around a C6711 DSP Starter Kit, a board centered on a TMS320C6711 floatingpoint DSP (Texas Instruments). This high-performance, low-cost board is responsible for acquiring and sampling eye movement data and subject responses, processing these data in real time, and monitoring real-time performance. It operates at $150 \mathrm{MHz}$, delivering 600 million floating-point operations and 1,200 million instructions per second. It includes $6 \mathrm{MB}$ of external SDRAM and $128 \mathrm{~KB}$ of external flash memory. Communication with the host PC occurs via a host port interface (HPI), a proprietary parallel port developed by Texas Instruments that provides access to DSP memory without interfering with ongoing processes.

As shown in Figure 4, the DSP board is coupled with two input/output interfaces: an ADS8364 evaluation module, a board that enables A/D conversion of six analog channels at a maximum sampling frequency of $250 \mathrm{KHz}$ with 16 bits of precision, and a custom-built digital interface designed to acquire subject responses from a Playstation 2 joypad (Sony Computer Entertainment). Both interfaces are connected to the DSP board via the 80-pin expansion daughter card interface. Subject responses and oculomotor signals are acquired through these $\mathrm{I} / \mathrm{O}$ interfaces. In the case of an analog eyetracker (as in the experiments described in this article) eye movement signals are sampled by the ADS8364 at a suitable sampling frequency.

The board also acquires the output signal produced by a fast-response photocell placed in a corner of the psychophysics monitor. This signal is used to detect the refreshing of the monitor and to check real-time compliance (see the Dropped Frame Detection Module subsection below).

\section{DSP Firmware}

A variety of routines run continually on the DSP board to control the flow and processing of input data and to

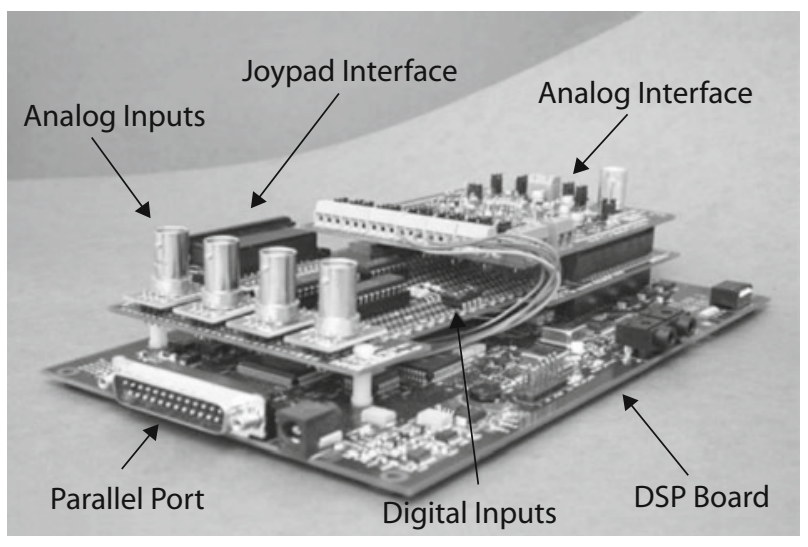

Figure 4. EyeRIS dedicated hardware. This device is built around a digital signal processor (DSP) equipped with analog and digital inputs. Communication with the host computer occurs via a parallel port. 
manage communications with the eyetracker, joypad, and host PC. This ensemble of routines makes up EOS, the "Eyetracker Operating System." EOS is based on DSP/ BIOS Version 2 (Texas Instruments), a scalable real-time kernel that requires no runtime license fee and enables clean partitioning of real-time applications. EOS routines that process incoming eyetracker data are organized into a processing pipeline, as shown schematically in Figure 5B. Data are processed serially by the various stages of the pipeline, each of which modifies or adds to the data, before forwarding the resulting packet to the next stage. The pipeline consists of the following three main stages.

Voltage-Angle Conversion Module. This first stage converts eye movement data recorded by the eyetracker into degrees of visual angle. Conversion occurs on the basis of a calibration procedure performed at the begin- ning of each experimental session. The calibration consists of two phases. In the first phase, the subject sequentially fixates on a grid of nine points equally spaced within the working area of the display. The transformation from eye movement coordinates to degrees of visual angle is determined by interpolation over these nine points (this procedure requires prior knowledge of the distance between the monitor and the subject and of the size of the display). Each fixation point is displayed for $5.5 \mathrm{sec}$, with the last $3.5 \mathrm{sec}$ of each fixation used to calculate the mean and variance of the gaze location. If the variance of the eye position exceeds a preset threshold, the fixation marker is redisplayed and the estimation procedure repeated. This initial rough calibration is followed by a fine-tuning stage in which the subject adjusts and/or confirms the transformation. In this stage, the subject uses the joypad to refine

A

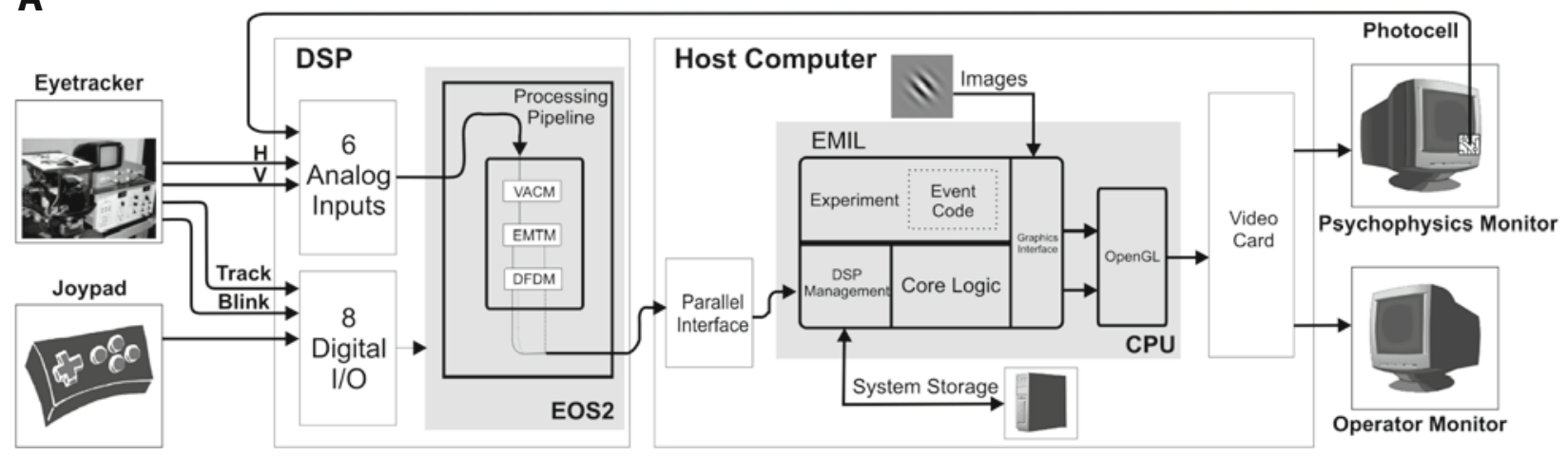

B
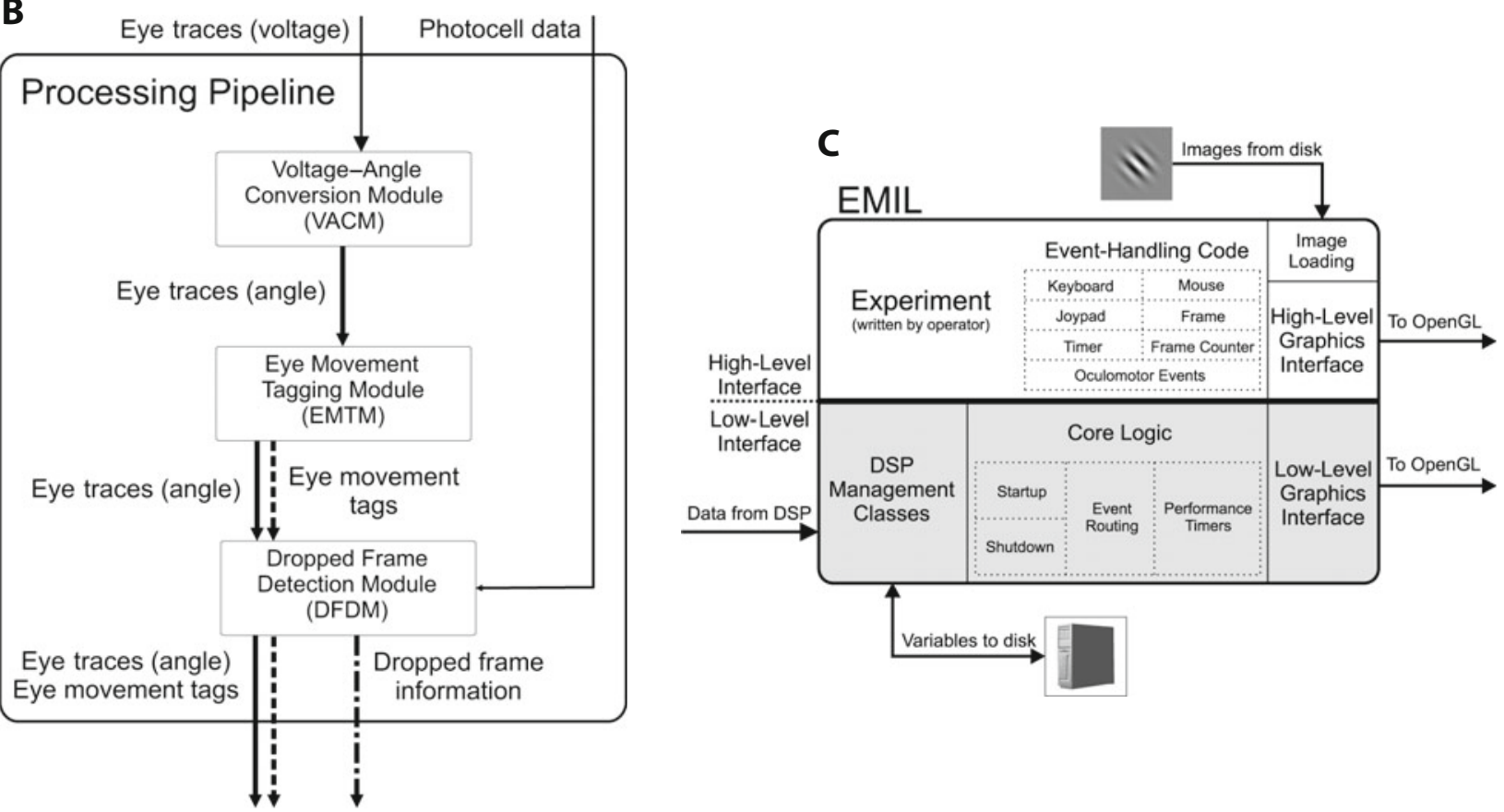

Figure 5. Detailed system architecture. (A) Processing occurs in parallel on the dedicated digital signal processor (DSP) board and on the host computer. (B) On the DSP, data pass through a processing pipeline that extracts variables relevant to the experiment. (C) On the host computer, the routines of the Eye Movement Integrated Library (EMIL) take care of system management tasks (lowlevel interface, shaded) and enable the user to specify the experimental protocol (high-level interface). See text for details. 
the transformation by comparing his/her direction of gaze, displayed in real time on the screen, against the same grid of fixation points. In the experiments described here, the voltage-to-angle transformation was performed by means of linear interpolation. This transformation, which takes only a minute portion of the overall pipeline processing time, was made possible by virtue of the highly linear behavior of the dual-Purkinje-image (DPI) eyetracker in the central region of the visual field. The final conversion from visual field angles to screen pixels is accomplished via a predefined routine that is part of the EyeRIS software interface.

Eye Movement Tagging Module (EMTM). This module is responsible for examining eye movement data, estimating motion variables, and detecting and characterizing predefined oculomotor events. To estimate eye movement velocity and acceleration, incoming traces of oculomotor activity are processed by a windowing technique based on the adaptive finite impulse response estimation algorithm (Janabi-Sharifi, Hayward, \& Chen, 2000). This method is designed to filter noise and minimize the error variance while maximizing estimation accuracy. During an experiment, the EMTM continually estimates kinematic signals and processes them according to rules specified by the experimenter to identify the desired categories of oculomotor events. Each of these events possesses parameters that are relevant to the particular EMCD experiment. The experimenter can modify any of the thresholds used to categorize eye movements at any time during an experiment. In the experiments described here, the EMTM used four predefined oculomotor events: saccade, microsaccade, drift, and fixation. In addition, the events saccade, microsaccade, and drift each possessed the associated kinematic parameters: amplitude, duration, and mean velocity. Categorization of the four events occurred on the basis of the following predefined rules. A saccade event was flagged any time all of the following three conditions based on user-defined criteria occurred: (1) the angular velocity of the eye exceeded a minimum event velocity threshold, (2) the duration of the event was longer than a minimum event duration threshold, and (3) the amplitude of the movement was greater than a minimum saccade amplitude threshold. Event parameters were evaluated upon completion of the event - that is, when the velocity returned below the minimum event velocity threshold. A microsaccade event was flagged any time that Saccade Conditions 1 and 2 occurred and the amplitude of the movement was greater than a given minimum microsaccade amplitude but smaller than the minimum saccade amplitude. Similarly, a drift event occurred when (1) the minimum event velocity threshold was not exceeded but (2) the eye movement velocity was greater than a preset minimum drift velocity threshold. Periods not labeled as saccade, microsaccade, or drift were labeled as fixation. The thresholds used in the experiments described in this article can be found in Table 1 .

Dropped Frame Detection Module (DFDM). Each component of EyeRIS has been designed and tuned to operate within a predetermined time window, so that the total time elapsed from the acquisition of eye movement data to the update of the stimulus on the display is less than the time required to draw two frames on the CRT display $(10 \mathrm{msec}$ at a refresh rate of $200 \mathrm{~Hz})$. Since EyeRIS is a general-purpose system in which EMCD procedures are specified by the experimenter, runtime information cannot be obtained a priori by means of a static analysis (i.e., the total number of operations required by the experiment for rendering the stimulus). As in every realtime system with hard deadlines, monitoring of runtime performance is therefore necessary to verify that time constraints are respected (Chodrow, Jananian, \& Donner, 1991; Tsai, Bi, Yang, \& Smith, 1996). To that end, EOS employs the DFDM, a dedicated stage of the processing pipeline, which provides nonintrusive real-time performance monitoring of the system. As shown in Figure 6, performance monitoring is accomplished by observing a small, flickering square displayed in a corner of the psychophysics monitor. This square alternates from black to white at every frame. This strong luminance oscillation is recorded by a photocell attached to the monitor and is processed by the DFDM to detect interruptions in the normal flickering of the square. The presence of a square with the same intensity value in consecutive frames indicates that one or more of the frames planned by the experimenter was not rendered in time. Therefore, the stimulus was not updated within the predefined time interval, and the realtime deadline was violated. Detection of a missed frame triggers a signal that is reported to the experimenter in case action is needed during the execution of the experiment. For example, the experimenter may decide to repeat or discard the trial in which the error occurred if the results are invalidated by time delays.

\section{Eye Movement Integrated Library (EMIL)}

The EMIL consists of an ensemble of $\mathrm{C}++$ routines running on the host computer that allow access to every element of EyeRIS. To simplify the task of writing experiments, EMIL is divided into two layers of functionality, as illustrated in Figure 5C. A collection of low-level classes is dedicated to handling the basic tasks necessary for the proper functioning of the system, such as communication and hardware management. In general, the experimenter does not need to deal directly with functions in these classes, because they are typically called by other functions. A high-level interface provides a simple yet powerful set of functions that the experimenter can use to design experiments. Since EMIL is made available as open-source software, users can freely share their code and progressively enrich the range of available functions.

Table 1 Eye Movement Tagging Module Default Thresholds

\begin{tabular}{ll}
\hline \multicolumn{1}{c}{ Feature } & \multicolumn{1}{c}{ Threshold } \\
\hline Minimum event velocity & $15 \mathrm{deg} / \mathrm{sec}$ \\
Minimum event duration & $20 \mathrm{msec}$ \\
Minimum saccade amplitude & $0.5 \mathrm{deg}$ \\
Minimum microsaccade amplitude & $0.1 \mathrm{deg}$ \\
Minimum drift velocity & $0.05 \mathrm{deg} / \mathrm{sec}$ \\
\hline
\end{tabular}




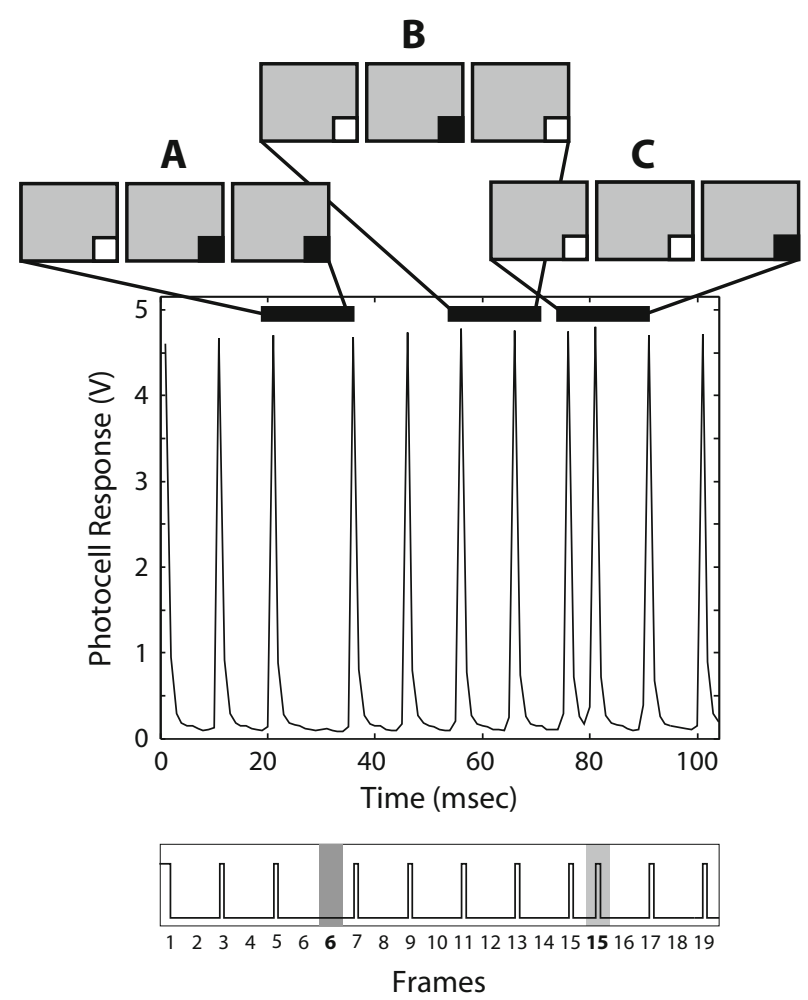

Figure 6. Monitoring of real-time performance. EyeRIS's realtime compliance is verified by monitoring the flickering of a small square in a corner of the display. The top panels represent successive frames of the psychophysics display. The signal recorded by the photocell (center graph) is processed by a dedicated module that checks for missing frames. The three marked intervals show the three possible cases: (A) prolonged presence of the black square (a white-square frame was missed), (B) regular flickering, or (C) prolonged presence of the white square (a black-square frame was missed).

Low-level software. EMIL's low-level interface consists of three main components. These components are responsible for handling basic system management and core logic, controlling EyeRIS dedicated hardware, and providing an interface for the system's graphics engine.

The first set of classes performs primary system management tasks, such as start-up and shutdown operations, initialization of display settings, and initiation and termination of communication between the host computer and the DSP. To avoid using computer resources that introduce indeterminate delays in the rendering, and to minimize the incursion of other Windows processes, the core logic unit makes extensive use of RAM and designates the execution of the experiment as a very high-priority process.

An extensive collection of core logic routines is responsible for implementing the mechanisms that underlie the execution of EMCD experiments. These routines respond to oculomotor events and make the corresponding oculomotor variables available to the high-level functions that implement the experiment. The core logic unit also maintains and responds to a number of high-precision timers that continuously monitor system performance. These timers give a breakdown of the relative distribution of time con- sumed by various operations in the code that implements an experiment. This detailed information is useful for the debugging and performance-tuning of experiments.

The second set of classes encapsulates all communication with and control of the DSP board. The tasks most frequently performed by these classes are the retrieval of oculomotor variables from the DSP board, the calibration of the DSP processing pipeline, and the buffering of eye traces and other variables for later file storage. These classes also implement an emulation mode, so that EyeRIS can operate without the DSP board. This feature is useful for replaying recorded experiments, analyzing stored data, and debugging new experiments.

The low-level graphics classes create an interface to OpenGL (Silicon Graphics), a graphics library that EyeRIS uses to render stimuli on the display. EMIL's highlevel graphics routines operate on top of this interface. Users with programming experience can use this interface to extend the range of stimuli that EyeRIS can display. All OpenGL routines, including extensions, are visible and accessible via this interface, granting tremendous flexibility and power over the design of stimuli.

High-level software. A collection of high-level functions provide a simple-to-use yet powerful software environment for designing EMCD experiments. A number of utility classes are provided for accessing input data and oculomotor signals. Other functions access the highprecision timers and the state variables of low-level routines. EMCD experiments are designed by customizing event-handling routines in a template that is provided with the library. This template includes well-documented points into which custom code can be inserted to handle all system events, including oculomotor events, keyboard strokes, and timed events.

The high-level software also includes routines that interface with all the primary functionalities of the system. For example, a single function implements the standard EyeRIS calibration procedures that are needed at the beginning of an experiment. Input/output functions allow stimuli to be loaded from files saved in standard graphics formats. Furthermore, functions are available that implement common EMCD protocols such as predefined gazecontingent motion of the stimulus, retinal stabilization, space-variant sampling, and the flashing of stimuli in response to oculomotor events.

\section{System Timing}

EyeRIS is designed to process data in real time and, at each frame rendered on the display, to update the stimulus on the basis of the most recent oculomotor data available. The delay between the occurrence of an oculomotor event and the stimulus update depends on the refresh rate of the display and the position of the stimulus on it. Since a video frame in a CRT is rendered as a series of horizontal lines from top to bottom, stimuli on the top portion of the screen are refreshed before stimuli on the bottom. At a refresh rate of $200 \mathrm{~Hz}$, EyeRIS's delay from the sampling of eye movement data to the visualization of the stimulus on the screen varies between 5 and $10 \mathrm{msec}$, with an average delay of $7.5 \mathrm{msec}$. 
EyeRIS's timing over one video frame at a refresh rate of $200 \mathrm{~Hz}$ is shown in Figure 7. The EyeRIS dedicated hardware and host computer operate asynchronously, each following a separate clock signal. Every $1 \mathrm{msec}$, the DSP samples the input data and passes them through the processing pipeline. The output of the processing pipeline is written into a buffer that the host computer can access without interfering with the DSP (the DSP thus does not need to suspend execution to communicate with the host computer). To achieve synchronization of events, acquisition of all analog input channels, including the photocell signal, is regulated by the same internal clock so that all samples are taken simultaneously. This synchronization enables EyeRIS to maintain both precise timing between oculomotor events and the update of the stimulus on the display and a complete knowledge of the stimulus displayed on the screen at every point of an eye movement trace.

Operations performed on the host computer are instead synchronized by the video card's vertical refresh interrupt. When this interrupt signals that the video card is ready to render a new frame, the computer contacts the DSP and reads all oculomotor events and variables generated during the previous frame. Communication and data transfer between the DSP and the host computer takes approximately $1.6 \mathrm{msec}$. When the psychophysics monitor is being refreshed at $200 \mathrm{~Hz}$, this leaves $3.4 \mathrm{msec}$ for processing and rendering the next video frame. On modern video cards, this time is sufficient for rendering an extremely wide range of complex stimuli, as demonstrated in the EMCD Benchmark section below.

If rendering takes more time than is allotted, the experiment misses the frame swap deadline, and a dropped frame occurs (see the Dropped Frame Detection Module subsection). The system is capable of graceful recovery from such interruptions, continuing at the next frame with a small backlog of samples to process. For most EMCD procedures, this backlog is not a burden, since only the most recent eye movement data are used to update the stimulus.

\section{RESULTS}

EyeRIS performance has been carefully evaluated in a variety of experimental tasks, and the system is now routinely used in our psychophysical experiments on fixational eye movements (Desbordes \& Rucci, 2004). To demonstrate EyeRIS's flexibility and robust design, this section first describes the system's computational performance and the accuracy of its real-time classification of eye movement events, then provides examples of the application of the system to visual psychophysics.

\section{EMCD Benchmark}

In a general-purpose system for EMCD control, the computational load imposed by any experiment depends on the EMCD procedures specified by the user. Therefore, one of EyeRIS's most critical requirements is that it have the capability of rendering a significantly large number of graphic objects while simultaneously processing and responding to oculomotor events in real time. In a series

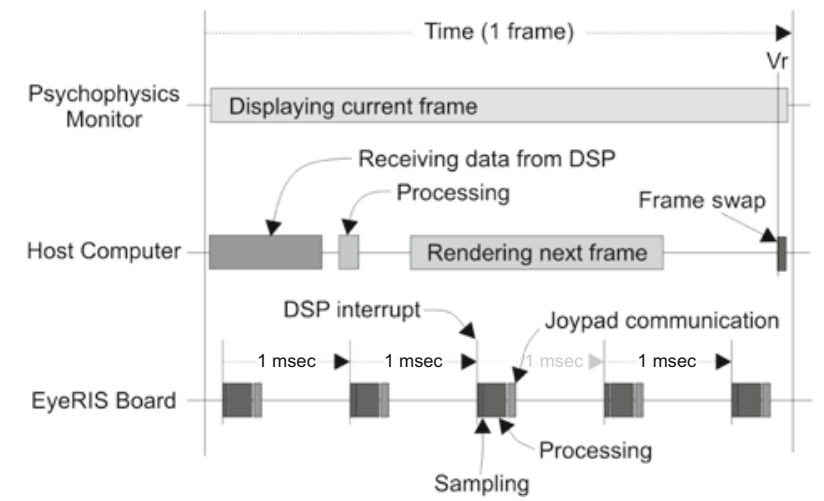

Figure 7. Real-time functioning of EyeRIS. The eyetracker data and subject responses are processed by dedicated EyeRIS hardware during a frame and transferred to the host computer before the next frame. While the digital signal processor (DSP) continues to sample and process input signals, the central processing unit and video card on the host computer use available data to appropriately modify the stimulus. The updated stimulus is then displayed at the next video frame. Note that DSP and host computer processing are not synchronized. "Vr" represents the vertical synchronization signal of the CRT monitor.

of tests, we estimated the number of graphic objects that EyeRIS can display and manipulate in a gaze-contingent manner without losing real-time compliance. The experiments were run at a refresh rate of $200 \mathrm{~Hz}(5-\mathrm{msec}$ frame time), the maximum frequency supported by our Vision Master Pro514 monitor (Iiyama, Inc.). Figure 8 shows the results of three of these experiments.

In the first experiment, we measured the number of solid, untextured planes (one of the graphics primitives of EMIL's high-level graphics interface) that can be moved simultaneously in real time according to the position of gaze. A function generator simulated eye movement signals. EyeRIS performance was evaluated in two ways: by examining the output of the DFDM, which continuously monitors real-time compliance in the DSP processing pipeline, and by means of the system's internal timers. These timers measure the intervals required by rendering and communication with the DSP board. Adherence to real-time constraints was also verified by direct inspection of photocell readings with a digital oscilloscope. The results of this experiment are shown in the top row of Figure 8. The lines in Figure 8B represent the average total time needed for updating the stimulus on the screen and the portion of this time consumed by communication between the EyeRIS hardware and the host computer. As expected, only the rendering time increased with the system load, whereas the communication interval was not affected by the number of planes displayed. As shown in Figure $8 \mathrm{~B}$, the average interval required to render each frame remained below $5 \mathrm{msec}$ while rendering up to $14,00010 \times 10$ pixel planes. Over this threshold, processing became too intensive, and real-time compliance was lost because the time required to render the new frame exceeded the interval between frame swaps.

The middle row of Figure 8 shows the results of a similar test using textured planes, another graphics primitive of 

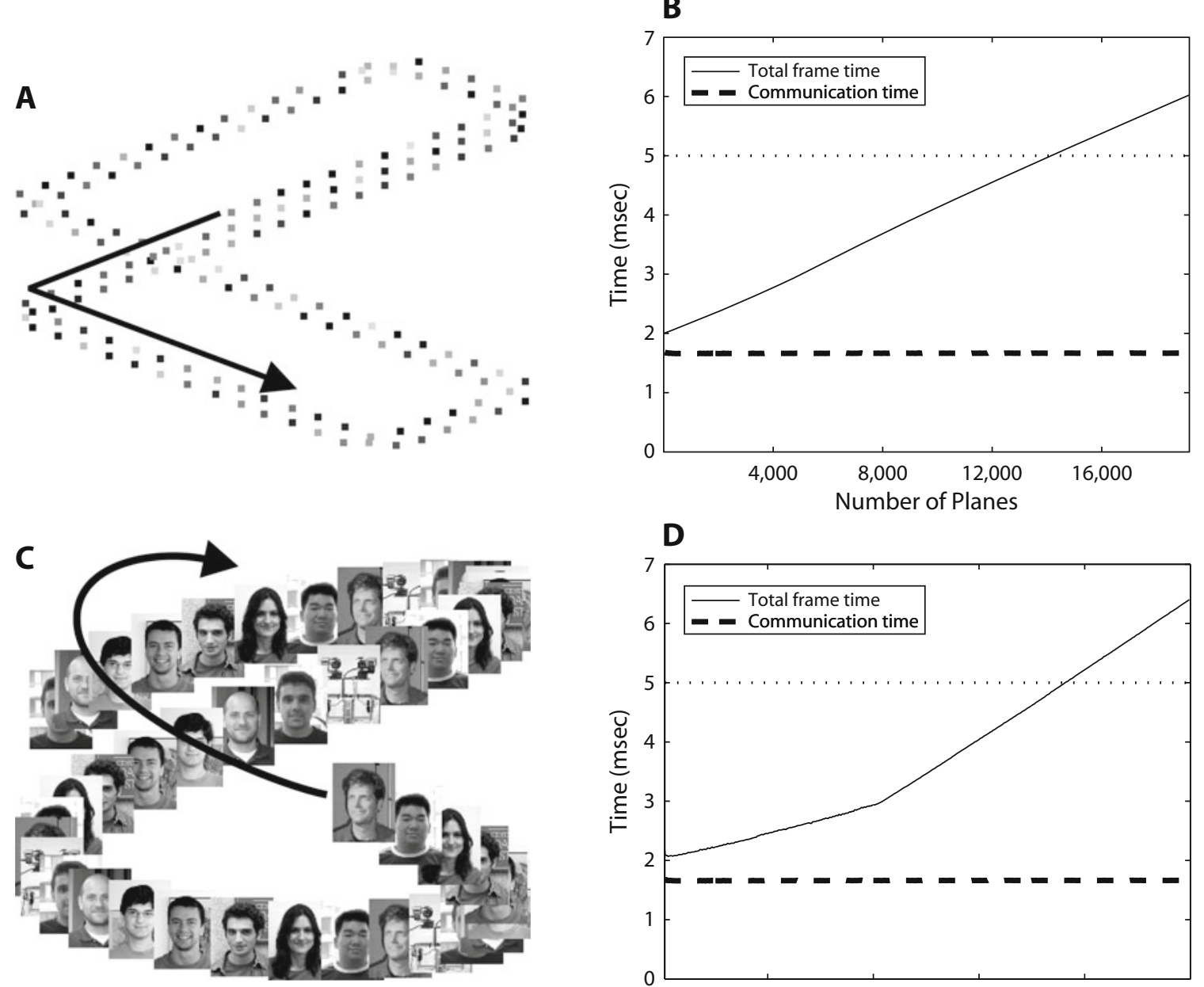

D

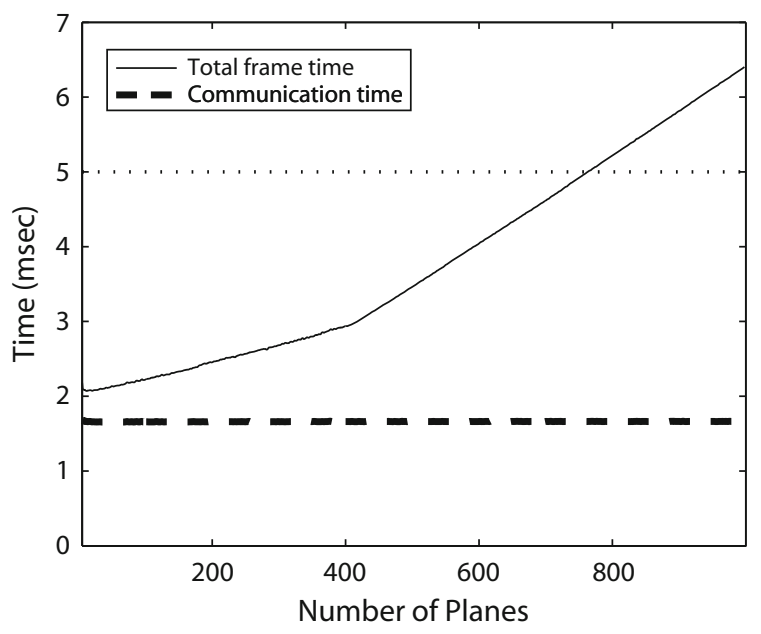

$\mathbf{E}$

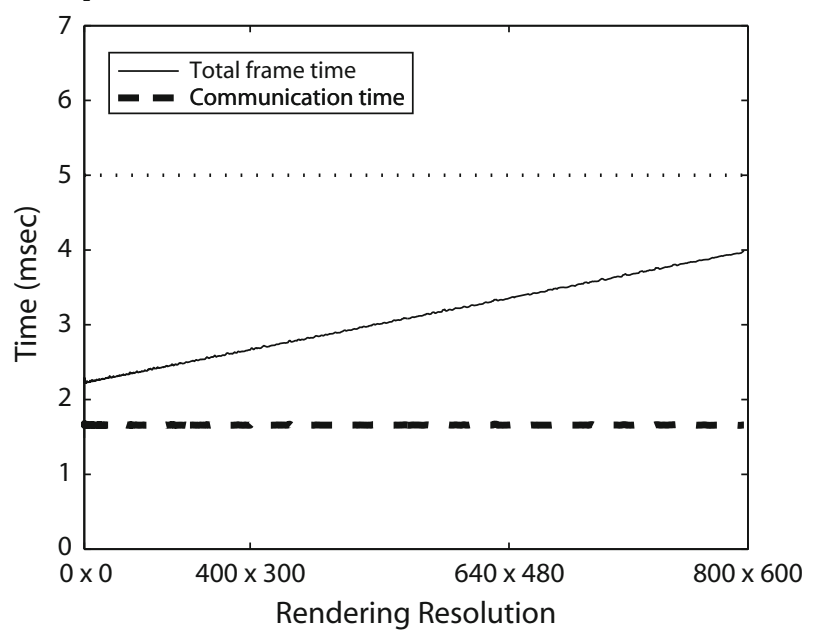

Figure 8. EyeRIS performance under stress tests designed to quantify the number of graphic objects that can be simultaneously manipulated in a gaze-contingent manner. Each row of panels shows the results of a different experiment. The left panel in each row presents an illustration of the experiment, and the right panel graphs the performance of EyeRIS in the experiment as complexity increases: (A) $10 \times 10$ pixel solid-color squares translated according to gaze position as simulated by a function generator. (C) Full 24-bit-color $100 \times 125$ pixel rectangles translated according to gaze position. (E) Space-variant sampling. On the right, panels B, D, and $F$ present the timing results for the test in the previous panel, indicating the number of planes at which the system lost real-time compliance. In each of panels B, D, and F, the dashed line represents the component of the frame calculation time consumed by communication with the DSP, and the solid curve is the total frame calculation time. 
EMIL's interface. Rendering of these planes is significantly more computationally intensive than that of untextured, solid-color planes. As the data show, EyeRIS successfully rendered and updated in a gaze-contingent manner 760 $100 \times 125$ pixel textured planes without losing real-time compliance. The results of these two experiments demonstrate EyeRIS's ability to handle a large number of graphics primitives that would allow for execution of a great variety of EMCD experiments. Indeed, it should be noted that a typical experiment would use only a small fraction of this rendering capacity. For example, the experiments described in the Examples of EMCD Experiments section never displayed more than two planes at once.

To give a concrete example of a useful EMCD procedure, in a third experiment, we measured EyeRIS's ability to maintain real-time compliance during space-variant sampling. Following the algorithm introduced by Perry and Geisler (2002), an image was resampled according to the position of gaze to mimic the resolution of the human retina (see Figure 8E). Figure 8F shows the results of this experiment as the number of pixels composing the image was gradually increased. As shown by the data, EyeRIS never lost real-time compliance during the test, even during full-screen rendering at $800 \times 600$ pixels, the maximum resolution allowed by the CRT monitor at a refresh rate of $200 \mathrm{~Hz}$.

\section{Eye Movement Classification}

Another serious challenge faced by a general-purpose system for EMCD control is the real-time categorization of oculomotor events. When examining eye movement traces, human experts typically base their categorization criteria on the local waveform of the signals around each event. For example, to determine whether ocular drift occurs at time $t$, a visual scientist would display the considered data segment on the computer monitor and evaluate the velocity both before and after $t$. A system that operates in real time, however, can perform categorization only on the basis of past data. To examine the accuracy of realtime categorization of oculomotor events, we compared the performance of our EMTM with the classification provided a posteriori by a human expert. This direct comparison shows that a number of fundamental oculomotor events can be reliably detected and labeled in real time.

Table 2 summarizes the results of real-time categorization of three different oculomotor events: saccades, drifts, and fixations. The eye movements of 2 subjects were sampled by means of a Generation 6 DPI eyetracker (Fourward Technologies) during presentation of images of natural scenes. Images were displayed for a total of $50 \mathrm{sec}$ of recording

Table 2

Results of Real-Time Categorization for Different Oculomotor Events

\begin{tabular}{lccc}
\multicolumn{4}{c}{ Different Oculomotor Events } \\
\cline { 2 - 4 } EMTM & \multicolumn{3}{c}{ Human Expert } \\
\hline Saccade (\%) & Drift (\%) & Fixation (\%) \\
Drift & 100 & 0 & 0 \\
Fixation & 0 & 99 & 1 \\
\hline
\end{tabular}

time. The subjects observed the stimuli monocularly, with the left eye covered by an eye patch. A headrest and bitebar were used to minimize head movement. The data in Table 2 compare the classifications made by the EMTM during the acquisition of eye movement traces with those manually performed by an expert after the oculomotor data were acquired and stored. Eye traces were individually analyzed, and the categorizations performed by EyeRIS were either confirmed or marked as incorrect. The percentages shown in Table 2 are relative to the numbers of events counted in the manual classification. As can be seen, the EMTM compares remarkably well with the judgments of a human expert, correctly identifying all saccades and deviating by only $1 \%$ in the cases of drifts and fixations.

\section{Examples of EMCD Experiments}

To provide examples of the application of EyeRIS in visual psychophysics, this section describes two EMCD experiments that we have used in our research on the visual functions of fixational eye movements (Desbordes \& Rucci, 2004; Rucci \& Desbordes, 2003). The experimental setup was the same one described in the section above.

Image fading. During periods of visual fixation, small eye movements, including small saccades and drifts, keep the image of the visual scene on the retina in constant motion (Ditchburn, 1955; Ratliff \& Riggs, 1950; Steinman, Haddad, Skavenski, \& Wyman, 1973). To investigate the visual function of this physiological motion of the retinal image, vision scientists have made ample use of retinal stabilization, an EMCD procedure that eliminates retinal image motion by translating the stimulus in a way that compensates for the subject's oculomotor activity (Ditchburn \& Ginsborg, 1952; Pritchard, 1961; Riggs \& Ratliff, 1952). As illustrated in Figures $2 \mathrm{C}$ and 2D, retinal stabilization has also found application in neurophysiology, where it has been used to reduce the variability of cell responses (Gur et al., 1997; Gur \& Snodderly, 1987; Snodderly et al., 2001). Unfortunately, the elimination of retinal image motion typically requires expensive and/or invasive systems, the accuracy of which critically depends on the careful execution of complex calibration procedures. As a consequence, a relatively small number of laboratories have systematically used retinal stabilization. EyeRIS provides a simple approach to retinal stabilization that can be adopted by many laboratories in visual psychophysics and neurophysiology.

It has been known since the 1960s that stimuli tend to fade or disappear in the absence of retinal image motion (Ditchburn \& Ginsborg, 1952; Riggs \& Ratliff, 1952). Indeed, image fading has often been used as a qualitative measure of the accuracy of retinal stabilization (Steinman \& Levinson, 1990). As part of our characterization of EyeRIS's performance, we used perceptual fading to compare the accuracy of retinal stabilization produced by EyeRIS with that of a stimulus deflector, a standard device for eliminating retinal image motion (Crane \& Clark, 1978). This device stabilizes the image on the retina by deflecting the field of view with mirrors that are directly driven by the eyetracker output signals.

In this experiment, 2 subjects reported the time at which complete fading of the stimulus occurred. Blocks of 15-20 
trials alternated between the two stabilization techniques, EyeRIS and the stimulus deflector. In both cases, the stimuli were single letters of the English alphabet, which varied randomly from trial to trial and covered $40^{\prime}$ of visual angle. Stimuli were displayed at the center of fixation within a uniformly gray background. Contrast levels were individually adjusted for each subject to slightly above his/her perceptual threshold. Both EyeRIS and the stimulus deflector were calibrated before each block of trials. Calibration of the stimulus deflector occurred as has been described in previous publications (Kelly, 1979; Rucci \& Desbordes, 2003). The EyeRIS calibration procedure is described in the DSP Firmware section.

Figure 9 summarizes the results of these experiments. Each graph shows data from 1 of the 2 subjects. For each subject, the two bars represent the average fading time obtained with each of the two methods of retinal stabilization. The results were evaluated over more than 100 trials in each condition. The actual number of trials differed among the various conditions, because trials in which saccades occurred were discarded from the data analysis to preserve a high quality of retinal stabilization. On average, fading times were $5.4 \pm 1.8 \mathrm{sec}$ (EyeRIS) and $5.5 \pm$ $2.4 \mathrm{sec}$ (stimulus deflector) for subject L.K. and 5.0 \pm $1.1 \mathrm{sec}$ (EyeRIS) and $5.4 \pm 1.6 \mathrm{sec}$ (stimulus deflector) for subject G.D. As these data show, the average fading times for stimuli stabilized by means of EyeRIS were statistically indistinguishable from those obtained with the stimulus deflector.

Stabilization after saccade. In addition to producing high-quality retinal stabilization, EyeRIS's unique combination of real-time processing of oculomotor signals and dynamic linking of oculomotor events to conditions for stimulus manipulation provides the basis for designing
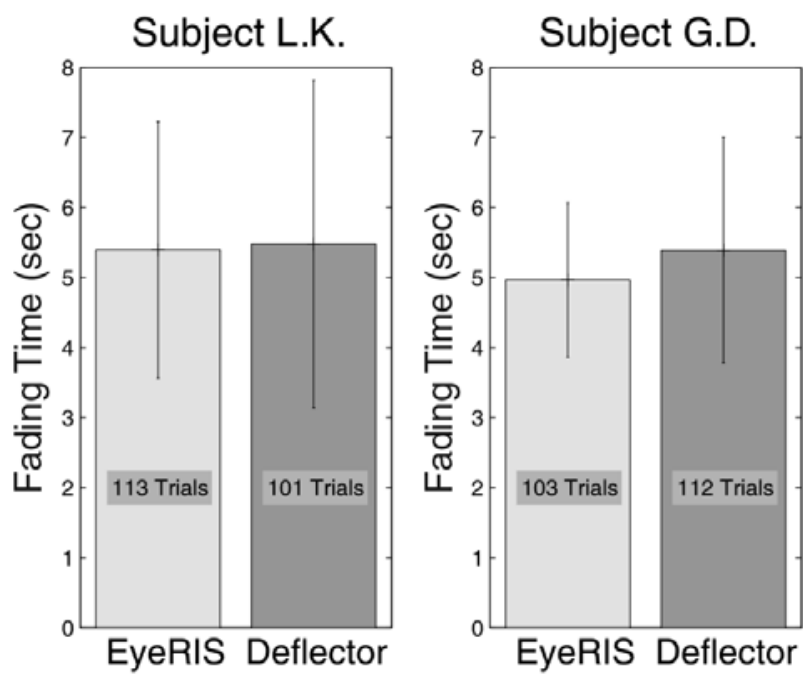

Figure 9. Comparing the accuracy of retinal stabilization provided by EyeRIS to that produced by the stimulus deflector, a standard device used to eliminate retinal image motion. The two graphs show the results for 2 different subjects. Each bar represents the average time required for the stimulus to fade, and error bars indicate standard deviations. experimental procedures that are not possible with other systems. One of the main limitations of standard methods of retinal stabilization is the requirement that the subject maintain steady fixation for prolonged periods of time. Since such methods do not allow switching between the two conditions of presence and absence of retinal image motion, fixation needs to be maintained for the entire duration of a block of trials. This abnormal viewing condition often prevents clear interpretation of the experimental results. EyeRIS does not suffer from this limitation, since it allows for retinal stabilization to be turned on and off at any moment during the execution of an experiment. This flexibility enables the isolation and selective elimination of the retinal image motion produced by different types of eye movements, a fundamental step in the study of the functions of these movements.

To provide an example of this capability, Figure 10 presents an experiment in which the stimulus was selectively stabilized on the retina during the period of visual fixation following an initial saccade. The time course of the experiment is schematically illustrated in Figure 10A. At the beginning of each trial, subjects performed a saccade that relocated the direction of gaze from the center of the screen to a randomly cued location. Presentation of the stimulus occurred at the very onset of visual fixation (i.e., as soon as EyeRIS detected the end of the saccade) and was contingent on the saccade landing within $30^{\prime}$ of the cued location. The stimulus was displayed for $1 \mathrm{sec}$ at the cued location under one of the two conditions: retinal stabilization or normal retinal motion. Trials randomly alternated between the two conditions.

Figure 10B shows an example of the activation of retinal stabilization. Eye movement traces are plotted together with demarcations designating the times at which EyeRIS (1) detected the end of the saccade and (2) activated retinal stabilization. For comparison, the offline estimation of the time at which the saccade ended is also shown. In this example, retinal stabilization was activated less than $15 \mathrm{msec}$ after the onset of visual fixation. Complete histograms of the delays between the onset of visual fixation and the activation of stabilization are shown in Figure 10C. The two panels show the results for 2 subjects. In each histogram, data represent the number of trials in which stabilization occurred with a specified delay from saccade end. The end of the initial saccade was manually estimated by analyzing offline the recorded eye traces. Over all trials and subjects, the mean delay with which retinal stabilization was activated was only $12 \mathrm{msec}$. This delay is equivalent to three frames at a refresh rate of $200 \mathrm{~Hz}$.

\section{CONCLUSIONS}

By enabling spatiotemporal manipulation of the stimulus according to the subject's oculomotor activity, the technique of EMCD control finds useful application in many areas of vision science. Unfortunately, the lack of general-purpose systems for EMCD experiments, combined with the technical difficulty of real-time processing and control, has prevented the widespread application of this methodology. EyeRIS offers a flexible, powerful, 
A

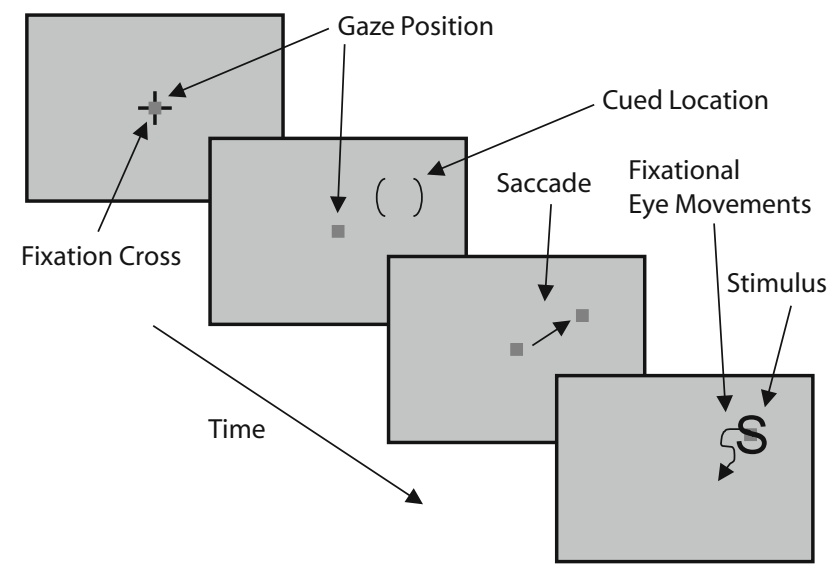

C

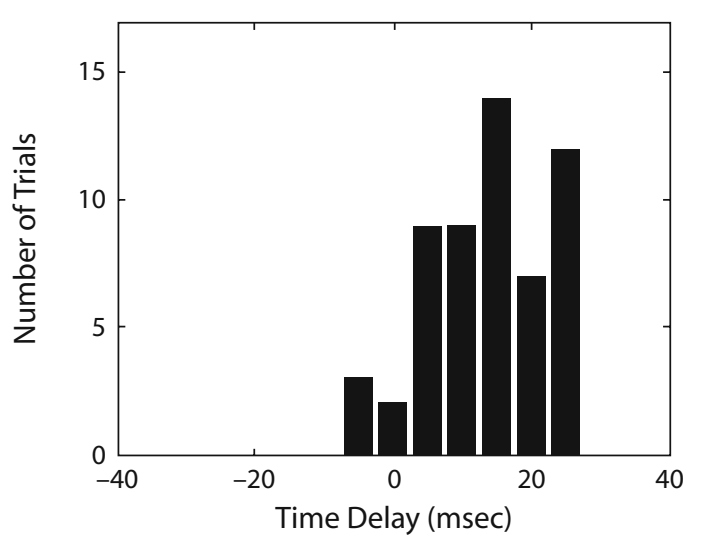

B
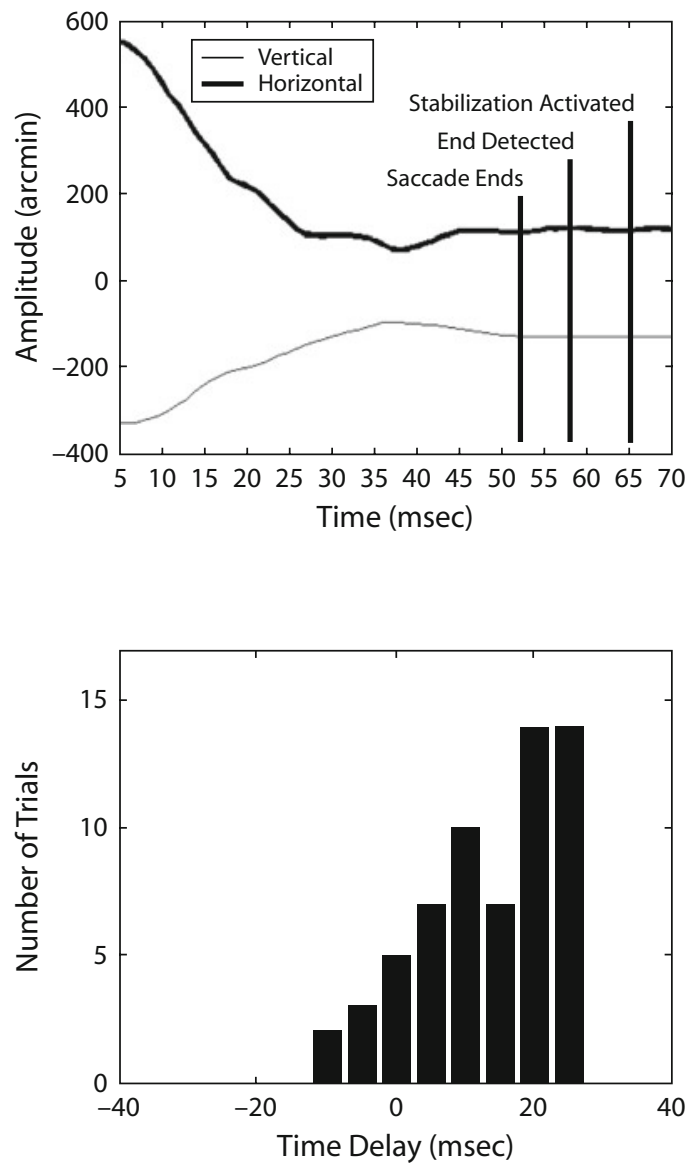

Figure 10. The stabilization-after-saccade experiment. (A) Experimental protocol. The gray dot indicates the subject's location of gaze. The stimulus was displayed for 1 sec after the end of the saccade if the center of gaze landed within the cued area. Trials could be randomly alternated between conditions of stabilization and of normal retinal motion. Arrows within the panels represent eye movements. (B) Example of detection of the end of a saccade. The two curves represent the horizontal and vertical components of eye movements, and the $x$-axis ticks mark when updates of the display on the CRT occur. The three vertical lines represent, respectively, the times at which the saccade ends, EyeRIS detects the onset of fixation, and retinal stabilization is activated. (C) Delay between the onset of visual fixation and the activation of stabilization. The two graphs show data for 2 different subjects.

yet cost-effective approach to the design and execution of EMCD experiments. By allowing the user to arbitrarily define oculomotor events and protocols for stimulus manipulation, EyeRIS enables a range of EMCD procedures that by far exceeds the capabilities of currently available systems.

Table 3 provides a comparison of EyeRIS's characteristics with those of other systems currently available to experimenters in the field of visual neuroscience. The other available systems vary greatly in their respective strengths and weaknesses and include both commercial products and free software. Public-domain systems, such as the open-source SVI Toolbox (Perry \& Geisler, 2002), can reach a wide circle of potential users, because they come at no cost and are simple to use. However, since they are based purely on software, these systems cannot reach the high refresh rates required by many experiments. Also, these kinds of systems generally lack monitoring of real-time compliance that could verify the integrity of the acquired data; that is, they do not guarantee that gazecontingent update of the stimulus occurs within a predetermined interval.

Systems that rely on dedicated hardware, as many commercial products do, generally operate at higher speeds and ensure real-time performance. However, these systems come at a high cost and tend to have very specialized functions. For example, the stimulus deflector (Fourward Technologies) can achieve high-quality retinal stabilization, but it does so at the expense of flexibility of experimental design (as described above in the Examples of EMCD Experiments section). Several manufacturers have recently expanded their products by introducing the possibility of EMCD manipulations in the control software. However, these systems are not specifically designed for EMCD control. For instance, in the EyeLink system (SR Research), data communication between the video 
Table 3

Comparison of EyeRIS With Other Available Systems

\begin{tabular}{|c|c|c|c|c|c|}
\hline System & Application & Realization & Refresh Rate & $\begin{array}{l}\text { Real-Time } \\
\text { Compliance }\end{array}$ & Cost \\
\hline SVI Toolbox & Foveated image & Software & up to $56 \mathrm{~Hz}$ & No & Public domain \\
\hline ViSaGe & $\begin{array}{l}\text { Stimulus generator, } \\
\text { retinal stabilization }\end{array}$ & Hardware/software & up to $160 \mathrm{~Hz}$ & Yes & $\$ 15,000$ \\
\hline EyeLink II & Gaze-contingent window & Hardware/software & up to $160 \mathrm{~Hz}$ & No & $\$ 36,000$ \\
\hline Stimulus deflector & $\begin{array}{l}\text { Retinal stabilization, } \\
\text { artificial scotoma }\end{array}$ & Optical/mechanical & 6-msec delay & N/A & $\$ 15,000$ \\
\hline EyeRIS & General purpose & Hardware/software & up to $200 \mathrm{~Hz}$ & Yes & $\$ 1,500^{*}$ \\
\hline
\end{tabular}

*Cost of the hardware components (eyetracker not included). The EMIL library is open source.

eyetracker and the host computer relies on Ethernet. This communication protocol adds an unpredictable delay, so the stimulus cannot be guaranteed to synchronize with the oculomotor signals. Similarly, the design of EMCD experiments with ViSaGe (Cambridge Research Systems), a board widely used in vision science laboratories, is hampered by various technical limitations. This system guarantees no frame loss during execution of the experiment, but it also does not allow any EMCD modification of the stimulus other than translation. As is schematically represented in Table 3, even though a number of gazecontingent stimulus manipulations are possible, the range of possible applications enabled by current systems remains limited. EyeRIS is the first general-purpose system for EMCD control ever developed.

In addition to a general characterization of EyeRIS performance, this article has reported examples of experimental applications related to our own research on fixational instability (Rucci \& Casile, 2005; Rucci \& Desbordes, 2003). In this context, we have shown that EyeRIS produces image stabilization with quality comparable to that of a stimulus deflector coupled with a DPI eyetracker, a standard noninvasive method for eliminating retinal image motion. This result is not surprising: The nominal delay of the stimulus deflector is $6 \mathrm{msec}$. At a refresh rate of $200 \mathrm{~Hz}$, EyeRIS updates a stimulus located in the central portion of the screen with a typical delay of $7.5 \mathrm{msec}$, which is comparable to the delay produced by the stimulus deflector. By enabling precise positioning of the stimulus on the retina, retinal stabilization has been shown to contribute to improving the efficiency of neurophysiological recordings and to decreasing the variability of neuronal response (Gur et al., 1997; Gur \& Snodderly, 1987). Based on the results shown here, EyeRIS could allow retinal stabilization to become a standard technique of behavioral neuroscience.

It is important to observe that the high quality of retinal stabilization produced by EyeRIS also comes with several important advantages with respect to the other methods currently available. A first critical advantage of EyeRIS concerns the flexibility with which retinal stabilization can be turned on and off. Other available stabilization methods do not allow any intermixing of trials in which the stimulus is stabilized on the retina with trials in which the same stimulus is viewed in the presence of the normal motion of the retinal image. For example, the stimulus deflector needs to be recalibrated every time it is turned on. Therefore, use of this device requires the execution of experiments in blocks of consecutive trials in which the stimulus is either stabilized or not. The subject is required to maintain steady fixation for the entire duration of the block of stabilized trials, a highly unnatural and difficult task. Because of these abnormal viewing conditions, the results of stimulus deflector experiments are often difficult to judge. EyeRIS does not suffer from this limitation. Since retinal stabilization with EyeRIS can be activated or deactivated at any time, trials in which the physiological motion of the retinal image is present or absent can be randomly alternated. This flexibility enables more natural viewing conditions as well as more accurate comparisons between the two conditions of retinal stabilization and normal retinal motion.

A second crucial advantage offered by EyeRIS is direct control over the quality of stabilization offered during the execution of the experiment. With any other stabilization technique, the experimenter has no way (other than using the feedback of the subject) to evaluate the quality of stabilization during the execution of an experiment. If a posteriori data analysis indicates that stabilization was not properly executed, the experiment needs to be run again. By showing the estimated location of gaze in real time on the experimenter's monitor, EyeRIS enables a direct check of the accuracy of retinal stabilization during the experiment. At any time during the experiment, the experimenter can examine the location of gaze estimated by EyeRIS while the subject maintains fixation on a point at a desired position on the psychophysics monitor. For this reason, highly experienced subjects are not required, and the reliability of subject judgment is not in question.

These advantages result directly from EyeRIS's design as a general-purpose system for EMCD control. EyeRIS allows flexible gaze-contingent control of the stimulus in both the temporal and spatial domains. The stabilizationafter-saccade experiment shown in Figure 10 provides an example of such precise temporal control. In this experiment, the stimulus was selectively stabilized during periods of visual fixation, whereas the retinal changes produced by macroscopic saccades were allowed to occur normally. This paradigm constitutes an important step in the study of the visual functions of fixational instability. Fixational eye movements are less frequent under the steady and prolonged fixation conditions required by 
other methods of retinal stabilization (Steinman \& Levinson, 1990). EyeRIS thus enables the study of the normal instability of visual fixation that occurs during the brief periods between saccades.

A similar level of gaze-contingent stimulus control is also available in the spatial domain. Whereas standard methods of retinal stabilization translate the entire visual field according to the subject's eye movements, EyeRIS is capable of stabilizing selected components of the visual scene. This function would be important, for example, during neurophysiological recordings, because it enables the experimenter to present a stationary fixation point on the screen while stabilizing on the retina the region covered by the receptive field of an electrophysiologically recorded cell. This operation is not easily accomplished with other systems.

Although the experimental examples given in this article refer to our own research, the general characterization of system performance and real-time detection of oculomotor events shows that EyeRIS can be applied to a wide variety of EMCD procedures. Indeed, the experimental possibilities offered by EyeRIS tremendously exceed those of any other available system. For example, in the study of attention, EyeRIS gives an unprecedented amount of control over the size and shape of the attentional window. Whereas other systems, such as the EyeLink II, allow only rectangular and circular apertures to move with the eyes (Pomplun, Reingold, \& Shen, 2001a), EyeRIS supports apertures of arbitrary shape. It is even possible to allocate multiple windows dynamically, a procedure useful in studies of divided attention. The system also enables the simple implementation of "hot spots," points in the display that trigger prespecified stimulus changes when the location of gaze falls on them. Furthermore, by allowing stimulus modifications on the basis of various parameters of oculomotor activity, EyeRIS opens the way for a new generation of EMCD experiments in which changes in the visual input depend only on the position of gaze.

Several measures were taken to ensure that vision science laboratories have easy access to EyeRIS. A critical design specification of EyeRIS is the capability of working with Windows. The use of this operating system is widespread among researchers in neurophysiology and psychophysics, who are often reluctant to undergo drastic changes in their experimental setups. All components of EyeRIS hardware are off-the-shelf boards that can be purchased directly from the manufacturer. The EyeRIS software is made available under an Open Source Initiative license and can be freely downloaded from the Internet. Users can also add their EMCD procedures to the EMIL library, thus enriching the range of EyeRIS applications and ensuring experimental reproducibility. By providing simple access to the methodology of EMCD control, EyeRIS may become a standard tool of laboratories that conduct research in visual psychophysics and neurophysiology.

\section{AUTHOR NOTE}

This material is based on work supported by the National Institutes of Health under Grant EY15732-01. We thank Max Snodderly for his use- ful comments on a previous version of the manuscript. Correspondence relating to this article may be sent to F. Santini, Department of Cognitive and Neural Systems, Boston University, 677 Beacon Street, Boston, MA 02215 (e-mail: santini@cns.bu.edu).

\section{REFERENCES}

Bertera, J. H. (1988). The effect of simulated scotomas on visual search in normal subjects. Investigative Ophthalmology \& Visual Science, 29, 470-475.

Chodrow, S., Jananian, F., \& Donner, M. (1991). Run-time monitoring of real-time systems. In Proceedings of the Twelfth International Real-Time Systems Symposium (pp. 74-83). New York: IEEE Press.

Crane, H. D., \& Clark, M. R. (1978). Three-dimensional visual stimulus deflector. Applied Optics, 17, 706-714.

Desbordes, G., \& RUCCI, M. (2004). Discrimination of briefly presented stimuli in the presence and absence of fixational eye movements [Abstract]. Journal of Vision, 4(8), 739a.

Ditchburn, R. W. (1955). Eye movements in relation to retinal action. Optica Acta, 1, 171-176.

Ditchiurn, R. W., \& GinsBorg, B. L. (1952). Vision with a stabilized retinal image. Nature, 170, 36-37.

Geisler, W. S., \& Perry, J. S. (1998). Real-time foveated multiresolution system for low-bandwidth video communication. In B. E. Rogowitz \& T. N. Pappas (Eds.), Human Vision and Electronic Imaging III (pp. 294-305). San Jose, CA: SPIE.

Gur, M., Beylin, A., \& SNODderly, D. M. (1997). Response variability of neurons in primary visual cortex (V1) of alert monkeys. Journal of Neuroscience, 17, 2914-2920.

Gur, M., \& SnOdDerly, D. M. (1987). Studying striate cortex neurons in behaving monkeys: Benefits of image stabilization. Vision Research, 27, 2081-2087.

Janabi-Sharifi, F., Hayward, V., \& Chen, C.-S. J. (2000). Discretetime adaptive windowing for velocity estimation. IEEE Transactions on Control Systems Technology, 8, 1003-1009.

Kelly, D. H. (1979). Motion and vision: I. Stabilized images of stationary gratings. Journal of the Optical Society of America, 69, 1266-1274.

Leopold, D. A., \& Logothetis, N. K. (1998). Microsaccades differentially modulate neural activity in the striate and extrastriate visual cortex. Experimental Brain Research, 123, 341-345.

Martinez-Conde, S., Macknik, S. L., \& Hubel, D. H. (2000). Microsaccadic eye movements and firing of single cells in the striate cortex of macaque monkeys. Nature Neuroscience, 3, 251-258.

MCCONKIE, G. W. (1997). Eye movement contingent display control: Personal reflections and comments. Scientific Studies of Reading, 1, 303-316.

Nikolov, S. G., Newman, T. D., Bull, D. R., Canagarajah, N. C., Jones, M. G., \& Gilchrist, I. D. (2004). Gaze-contingent display using texture mapping and OpenGL: System and applications. In Proceedings of the Symposium on Eye Tracking Research and Applications (pp. 11-18). New York: ACM.

Peli, E., \& Peli, T. (1984). Image enhancement for the visually impaired. Optical Engineering, 23, 47-51.

Perry, J. S., \& GeIsLER, W. S. (2002). Gaze-contingent real-time simulation of arbitrary visual fields. In B. E. Rogowitz \& T. N. Pappas (Eds.), Human Vision and Electronic Imaging VII (pp. 57-69). San Jose, CA: SPIE.

Pomplun, M., Reingold, E. M., \& Shen, J. (2001a). Investigating the visual span in comparative search: The effects of task difficulty and divided attention. Cognition, 81, B57-B67.

Pomplun, M., Reingold, E. M., \& Shen, J. (2001b). Peripheral and parafoveal cueing and masking effects on saccadic selectivity in a gaze-contingent window paradigm. Vision Research, 41, 2757-2769.

Pritchard, R. M. (1961). Stabilized images on the retina. Scientific American, 204, 72-78.

Ratliff, F., \& RigGs, L. A. (1950). Involuntary motions of the eye during monocular fixation. Journal of Experimental Psychology, 40, 687-701.

RAYNER, K. (1997). Understanding eye movements in reading. Scientific Studies of Reading, 1, 317-339.

Reingold, E. M., Loschky, L. C., McConkie, G. W., \& Stampe, D. M. 
(2003). Gaze-contingent multiresolutional displays: An integrative review. Human Factors, 45, 307-328.

RiggS, L. A., \& RATLifF, F. (1952). The effects of counteracting the normal movements of the eye. Journal of the Optical Society of America, 42, 872-873.

RUCCI, M., \& CASILE, A. (2005). Fixational instability and natural image statistics: Implications for early visual representations. Network, 16, 121-138.

Rucci, M., \& Desbordes, G. (2003). Contributions of fixational eye movements to the discrimination of briefly presented stimuli. Journal of Vision, 3, 852-864.

SNOdDERLY, D. M., Kagan, I., \& GuR, M. (2001). Selective activation of visual cortex neurons by fixational eye movements: Implications for neural coding. Visual Neuroscience, 18, 259-277.

Steinman, R. M., Haddad, G. M., Skavenski, A. A., \& Wyman, D. (1973). Miniature eye movement. Science, 181, 810-819.
Steinman, R. M., \& Levinson, J. Z. (1990). The role of eye movement in the detection of contrast and spatial detail. In E. Kowler (Ed.), Eye movements and their role in visual and cognitive processes ( $\mathrm{pp} .115-$ 212). Amsterdam: Elsevier.

TANG, J., Kim, J., \& Peli, E. (2004). Image enhancement in the JPEG domain for people with vision impairment. IEEE Transactions on Biomedical Engineering, 51, 2013-2023.

Toufaili, F. M., Seibel, E. J., \& McIntyre, D. J. (2004). Virtual vision loss simulator. Studies in Health Technology \& Informatics, 98, 388-390.

Tsai, J. J. P., BI, Y., YAng, S. J. H., \& Smith, R. A. W. (1996). Distributed real-time systems: Monitoring, visualization, debugging, and analysis. New York: Wiley.

(Manuscript received December 16, 2005; revision accepted for publication February 28, 2006.) 